ISSN 2073-4360

www.mdpi.com/journal/polymers

Review

\title{
Characterizations of Polyamidoamine Dendrimers with Scattering Techniques
}

\author{
Xiangyu Wang ${ }^{1}$, Ludovic Guerrand ${ }^{1}$, Bin Wu ${ }^{1}$, Xin Li ${ }^{1}$, Lauren Boldon ${ }^{1}$, Wei-Ren Chen ${ }^{2,3}$ \\ and $\operatorname{Li~Liu}{ }^{1, *}$
}

1 Department of Mechanical, Aerospace, and Nuclear Engineering, Rensselaer Polytechnic Institute, 110 8th Street, Troy, NY 12180, USA; E-Mails: wangx13@rpi.edu (X.W.); guerrl2@rpi.edu (L.G.); wub2@rpi.edu (B.W.); boldol@rpi.edu (L.B.); liznlixin@gmail.com (X.L.)

2 Soft Matter Thrust, Neutron Scattering Science Division, Oak Ridge National Laboratory, Oak Ridge, TN 37831, USA; E-Mail: chenw@ornl.gov

3 Department of Chemical \& Biomolecular Engineering, the University of Tennessee, Knoxville, TN 37996, USA

* Author to whom correspondence should be addressed; E-Mail: liue@ @rpi.edu;

Tel.: +1-518-276-8592; Fax: +1-518-276-2623.

Received: 20 December 2011; in revised form: 20 January 2012 / Accepted: 10 February 2012 /

Published: 21 February 2012

\begin{abstract}
Dendrimers are highly branched, open, covalent assemblies of branch cells (monomers) radially attached to a core in successive layers or generations. Major types of dendrimers include polyamidoamine, polypropylenimine, multiple antigen peptide, chiral, and Fréchet-type dendrimers. Their structure and dynamics can be explored by various techniques, such as scattering, spectrometry, and microscopy techniques. Specifically, the scattering techniques include small-angle neutron scattering (SANS), quasi-elastic neutron scattering (QENS), small-angle X-ray scattering (SAXS), and light scattering. Examples of their properties that can be explored by scattering techniques include: inter-molecular structure, intra-molecular cavity, radius-of-gyration (RG), hydrodynamic radius (RH), molecular weight, effective charge number of a single dendrimer molecule, water penetration into the interior of the dendrimers, and the internal dynamics. Of these properties, the hydrodynamic radius and molecular weight may be explored by DLS; the internal dynamics of dendrimers may be studied by QENS; and the others may be explored through SAXS and SANS. During the past several years, SANS and QENS have been used to study the structural properties and internal dynamics of various generations of polyamidoamine
\end{abstract}


dendrimers (PAMAMs). Their potential prospects as anticancer polymer drug carriers are also discussed.

Keywords: dendrimers; scattering techniques; anticancer

\section{Introduction}

Dendrimers are a family of nano-sized symmetric three-dimensional macromolecules with a tree-like structure consisting of the core, branches, and terminal groups. Over the past few decades, dendrimers have garnered much attention from researchers in different areas because of their promising practical applications. In medical applications, for example, dendrimers (in the range of 1 to $100 \mathrm{~nm}$ ) are less prone to reticulum and endothelium uptake, and as a result behave more similarly to different important biological polymers [1]. Additionally, a near total monodispersity and an increase in density as a function of generation numbers allow for greater void space near the core, making room for potential drug molecules. Furthermore, multiple terminal functional groups can be synthesized, making a particular dendrimer more polyvalent or more specific. These features make dendrimers well-suited for the carriage of drugs to be delivered either at precise locations (site of a tumor) or over a predetermined amount of time. Dendrimers can also be used to emulate certain biological proteins such as insulin and hemoglobin [2].

\section{Basic Properties of Dendrimers}

\subsection{Basic Features}

Understanding the role of dendrimers in cancer treatment and diagnosis requires knowledge of their basic properties and applications. Their unique architecture and properties place them in a different class of polymers - dendritic polymers [3]. Dendrimers are highly branched, open covalent assemblies of branch cells (monomers) radially attached to a core in successive layers or generations [4]. Figure 1 below depicts a typical schematic of the structure.

Their architecture rests on three main components: an initiator core, the branch cell amplification region, and finally the outer terminal functional shell [3]. The core is often described as the information center of the molecule and ultimately defines the characteristics of the dendrimer. The amplification region determines the features of the bonds between the dendrimer and guest molecules through the void density and composition of the branch cells. Finally, the exterior layer represents the outer surface of the last shell and is composed of terminal functional groups used for various purposes, such as the foundation for subsequent shells or generations and the regulation of the flow of guest molecules into and out of the dendrimer [5]. It is worth noting that a dendrimer is not limited to spherical shapes but may also be found as a disk. 
Figure 1. Schematic Diagram of a typical Dendrimer [6] (Copyright Wiley-VCH Verlag $\mathrm{GmbH} \& \mathrm{Co} . \mathrm{KGaA}$. Reproduced with permission).

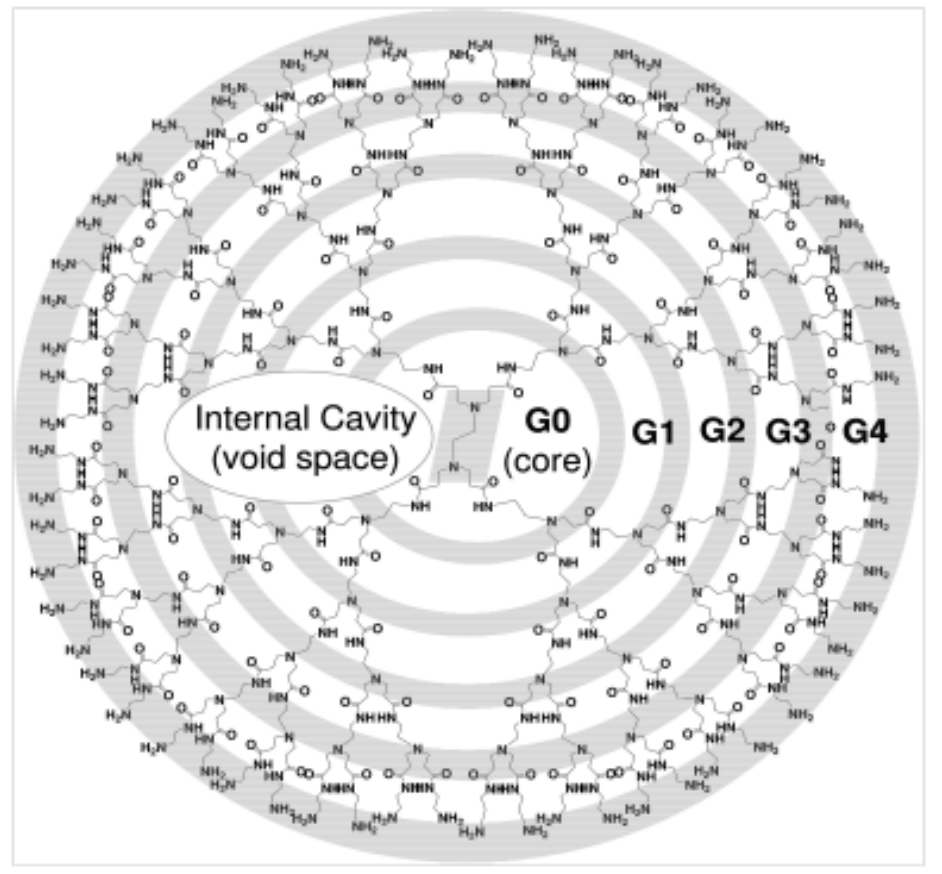

\subsection{Comparison with Conventional Synthetic Polymers}

More insight on the unique nature of dendrimers can be gained by exploring how they differ from other "conventional" synthetic polymers categorized as linear, cross-linked, and branched. While all of these types of basic monomeric blocks instill a large variety of properties, the arrangement of the blocks differs greatly. Dendrimers show very little branching or crosslinking and are almost entirely characterized by their hyperbranched structure [5]. These architectural differences have significant effects on their physical and chemical properties even when these polymers are composed of comparable monomer building blocks. In contrast to linear polymers, dendrimers show predictable shape variations instead of random coil configurations, occupy less hydrodynamic volumes, and have very low polydispersity. Dendrimers are also non-crystalline, amorphous substances exhibiting no repetition and a Newton type rheology with a lower viscosity that reaches a plateau at the fourth generation before declining [6]. Higher solubility is achieved along with isotropic electronic conductivity. More subtle differences can be observed when considering other dendritic structures. For instance, a constant density changes as a function of generation number for dendrimers with asymmetric branch cell arrangements; whereas, symmetrical branch cell arrangements achieve a minimum density between generations 4 and 7. Dendrimers are also unique among other polymers, because they experience exponential growth of the terminal functional group and maintain consistent shape as a function of generation number [5]. Finally, dendrimers can be considered the link in properties and architecture between thermoset and thermoplastic polymers [5]. 


\subsection{Comparison with Proteins (Biological Polymers)}

The functionalities and characteristics of dendrimers logically lead to a comparison with biological polymers or proteins. While only a few examples of dendrimer architectures are found in nature where the principal equivalent would be glycogen (involved in the storage of energy) and proteoglycans (involved in the absorption of energy), several similarities can be noted [5]. Both types are globular (when comparing to globular protein types), comparable in sizes, and composed of defined monomeric units arranged in a distinct architecture. Moreover, as is often the case with globular proteins, precise void space created by the folding of peptic chains afford these molecules special capabilities that can be imitated by the dendrimers' ability to host microenvironments within branch cells with properly designed active sites [7]. Furthermore, through non-covalent forces, dendrimers can exhibit a "lock and key" chemical behavior familiar to many proteins. Although many parallels were drawn between proteins and dendrimers a few differences should be mentioned. Dendrimers are more customizable through their synthesis and can be designed to achieve a wide variety of goals. Indeed, because of their architecture (radially attached branch cells), a greater surface area is available for the terminal functional groups. These terminal branches become less flexible with an increase in generation number due to crowding of the outer layer [5].

\subsection{Poly(amidoamine) Dendrimers (PAMAM)}

In 1985 Tomalia et al. [8] first discovered PAMAM dendrimers. They are the first family of dendrimers that have been synthesized and commercially available. PAMAM dendrimers are synthesized starting from an alkyldiamine core reagent. By reacting with the Michael addition of methylate, the smallest PAMAM dendrimers could be obtained [8]. Higher generations of PAMAM could be synthesized by a reaction of the small dendrimers with a large excess of ethylenediamine. Starting from G7, the de Gennes dense packing effect constrains the growth of dendrimers [9]. The growth of PAMAM dendrimers with generations higher than G10 is inhibited. Various generations of PAMAM dendrimers have specific size and shape characteristics. Their molecular weights are normally narrowly distributed with a high molecular uniformity. Their surface functional groups are ideal for potential chemical and biological reactions and applications [10].

\section{Characterization of Dendrimers}

\subsection{Characterization of Dendrimers by Small-Angle Neutron Scattering (SANS)}

The structural properties of dendrimers to be explored by SANS include: inter-molecular structure, intra-molecular cavity, radius-of-gyration $\left(\mathrm{R}_{\mathrm{G}}\right)$, effective charge number of a single dendrimer molecule, and water penetration into the interior of the dendrimers.

The structural information of SANS experiments is obtained by the comparisons between the experimentally detected spectra and the theoretical results of the system. The theoretical results were calculated with a reasonable effective potential $V_{e f f}(r)$ developed based upon statistical mechanics models such as the Ornstein-Zernike (OZ) integral equation theory. Neutrons scatter heavily (stronger signals from the experimental result) with the proton nucleus in the experimental system. They also 
scatter almost evenly with the other nucleus in the system. Neutron scattering has the unique capability of determining structural information of certain parts of the system through labeling (e.g., deuterating and/or protonating some parts of the dendrimers).

It is important to realize that neutron scattering experiments prefer the use of the solutions of $\mathrm{D}_{2} \mathrm{O}$ over $\mathrm{H}_{2} \mathrm{O}$. This is due to the fact that hydrogen has such a large cross section, and the scattering signals from the hydrogen in $\mathrm{H}_{2} \mathrm{O}$ molecules will cover up most contributions from the macromolecules. However, for a macromolecular solution, while substituting the $\mathrm{D}_{2} \mathrm{O}$ solvent for the $\mathrm{H}_{2} \mathrm{O}$ solvent at a condition $\left[H^{+}\right]=\left[D^{+}\right]$, the $\mathrm{pH}$ of a $\mathrm{H}_{2} \mathrm{O}$ solution is different from the corresponding $\mathrm{pD}$ in a $\mathrm{D}_{2} \mathrm{O}$ solution. It is generally agreed that $\mathrm{pD}=\mathrm{pH}+0.41$ [11]. Furthermore, the concentration of deuteron in solution is corrected by the measured deuteron activity [12]. The concentration of added acid used to protonate the amino groups, such as deuterium chloride, is represented by the acidity scale factor $\alpha$, which is defined as the molar ratio of acid to primary amine terminal groups:

$$
\alpha \equiv \frac{[\text { Acid }]}{\left[-\mathrm{NH}_{2}\right]}=\frac{[\text { Acid }]}{2^{n+2}[\text { PAMAM }]}
$$

where $n$ is the dendrimer generation. The word "protonation" is used to describe the binding between the deuterons and the amino groups. For typical experiments, the dendrimers were dissolved in $\mathrm{D}_{2} \mathrm{O}$ solvent at different levels of molecular protonation.

For the data analysis, models for the SANS absolute intensity $I(Q)$ have been developed [13,14] for dendrimer study, where the scattering intensity, $I(Q)$ is a function of the momentum transfer in reciprocal space, the scattering vector $Q=4 \pi \sin \left(\frac{\theta}{2}\right) / \lambda . Q$ is a scalar connected to the scattering angle, $\theta$, and the wavelength of the radiation, $\lambda . Q$ is at the range of $10^{-3}-1 \AA^{-1}$ in a typical SANS experiment with a selected sample-to-detector distance. The size of the region investigated in real space is related to $Q$ by $r=2 \pi / Q$, and falls in the range of 3-12 $\AA$ [15]. $I(Q)$ depends on the atomic features and the position of the atomic scattering centers [16]. Normally the predicted theoretical intensity distribution $I_{\text {model }}(Q)$ is utilized to fit with the SANS measured coherent scattering intensity $I_{\text {exp }}(Q)$. Results from the fitting process can be used to compute key parameters related with the structure of macromolecules, such as radius of gyration.

The SANS measured coherent scattering intensity $I_{\text {exp }}(Q)$ is corrected for detector background and sensitivity and for scattering contributed from empty cells. It is placed on an absolute scale according to a standard procedure described in $[13,14,17,18]$. Thereafter, $I_{\exp }(Q)$ is modeled by the following integral equation:

$$
I_{\text {exp }}(Q)=\int \frac{I_{\text {model }}(Q)}{\sqrt{2 \pi \delta(Q)^{2}}} \exp \left[-\frac{\left(z-Q_{m}\right)^{2}}{2 \pi \delta(Q)^{2}}\right] d z
$$

where $I_{\text {model }}(Q)$ is the theoretical intensity distribution, $\delta(Q)$ is the width of the experimental resolution function at $Q$, and $Q_{m}$ is the mean $Q$ value. The structural information of dendrimers could be obtained by the comparisons between the experimental spectra of $I_{\text {exp }}(Q)$ and the theoretical results $I_{\text {model }}(Q)$. The theoretical results were calculated with the commonly used monodispersed centrosymmetric core-shell model with diffusive interfaces [19] developed with statistical mechanics models such as the Ornstein-Zernike (OZ) integral equation theory. The integrity of the structural 
information provided by these theories was validated by MC or MD computational simulations [20]. $I_{\text {model }}(Q)$ can be further calculated by the so-called factorization approximation $[17,21]$ :

$$
I_{\text {model }}(Q)=A P(Q) S(Q)+I_{I N C}
$$

where $A$ is the scattering contrast between dendrimer particles and the solvent depending on the contrast, particle number density, and the particle volume, $P(Q)$, the normalized intra-dendrimer structure factor, and $S(Q)$, the inter-dendrimer structure factor. By assuming a diffuse interface between the solvent and dendrimer, $P(Q)$ is given by the modified fuzzy ball model which approximates the PAMAM molecular density profile by the convolution of a hard sphere with radius $R$ and a Gaussian with variance $\sigma^{2}$ characterizing the soft shell region [13,22,23], and $S(Q)$ is obtained by numerically solving the $\mathrm{OZ}$ integral equation for one component system in combination with the hypernetted chain (HNC) closure [24]. The effective inter-dendrimer interaction is approximated by the screened Coulombic potential and the essential physical quantities characterizing the charged PAMAM solutions, such as the effective charge carried by a single PAMAM dendrimer and the ionic strength, which can be converted from the fitted potential parameters based on the generalized one-component macroion approach (GOCM) [25]. In this approach, the radius of gyration $R_{G}$ can be calculated and expressed as a simple analytical function of $R$ and $\sigma$ :

$$
R_{G}=\sqrt{\frac{3}{10}\left(2 R^{2}+5 \sigma^{2}\right)}
$$

The normalized radial intra-molecular density distribution function, $\rho(r)$, is directly calculated by $R$ and $\sigma$ during calculation of $P(Q)$. The pair correlation function, $g(r)$, which gives the total correlation function between two given dendrimer molecules, may be calculated by $S(Q)$ through definition: $S(Q)-1 \propto$ FT $[g(r)-1]$ (FT: Fourier transform). Moreover, $S(Q)$ can lead to effective charge number of a single dendrimer molecule, $C_{\text {eff }}$ [13]. Thus, fitting $I_{\exp }(Q)$ with theory calculated $I_{\text {model }}(Q)$ will allow us to at least extract the following parameters: $P(Q), S(Q), R_{G}, \rho(r), g(r)$, and $C_{\text {eff }}$.

Figure 2. The experimental small-angle neutron scattering (SANS) coherent intensities I(Q) (symbols) obtained from the aqueous solutions of polyamidoamine dendrimers (PAMAM) dendrimers of three different protonated levels (alpha $=0,1$, and 2) at the concentration of $0.025 \mathrm{mg} / \mathrm{mL}$ at ambient temperature and the corresponding model fitting curves (Copyright IOP PUBLISHING. http://iopscience.iop.org/0953-8984/24/6/064116).

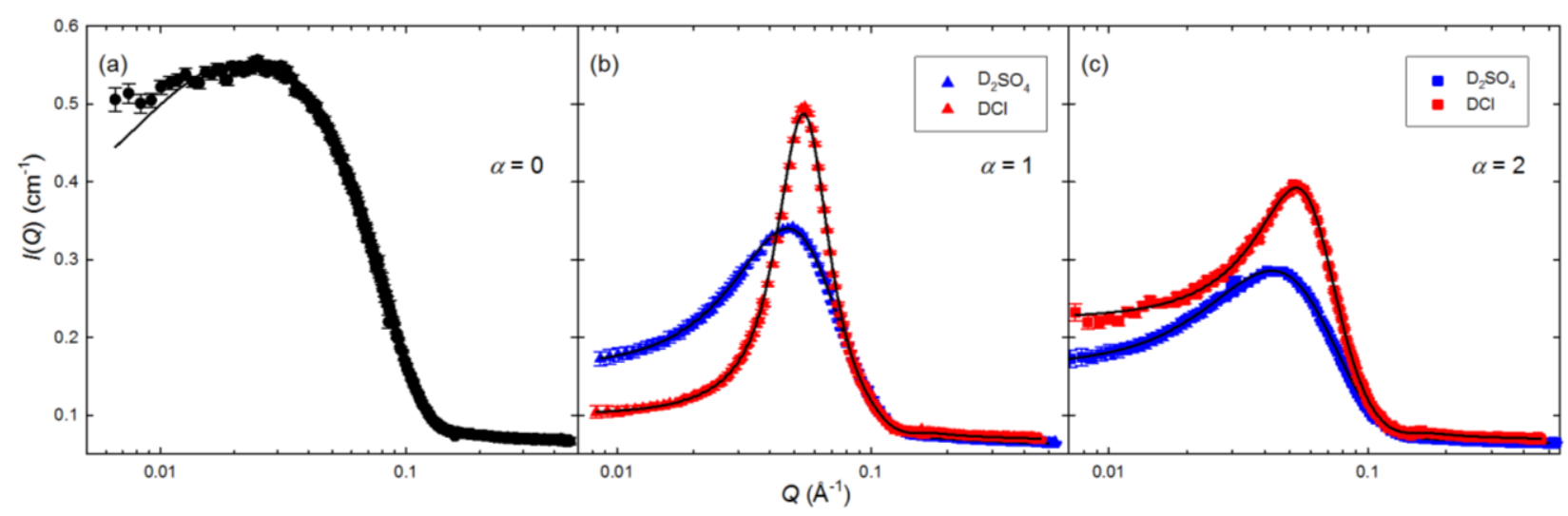


Figure 2 presented Liu et al.'s work [26] for this data analysis methodology, which is an experiment for PAMAM dendrimers at three different levels of $\mathrm{pH}$ values (molecular protonation levels $\alpha=0$ (Figure 2(a)), 1 (Figure 2(b)), and 2 (Figure 2(c))) and two different acids (blue symbols: deuterated sulfuric acid, and red symbols: deuterium chloride). It shows the good agreement between SANS intensities and model fitting results.

By using the SANS data analysis methodology, Chen et al. [13] studied the SANS results of charged G4 dendrimer solutions in various thermodynamic states by inter-dendrimer structure factor $\mathrm{S}(\mathrm{Q})$ and the intradendrimer structure factor $\mathrm{P}(\mathrm{Q})$. They proposed an instructive explanation about the counterions' effect on the conformation and structure of dendrimers and illustrated the effect of $\mathrm{pD}$ on the conformation of PAMAM dendrimers. They found that the number of counterions connecting with dendrimers increased first with the addition of $\mathrm{DCl}$, while the effective charge remained invariant with a further increase of molecular protonation. Based on SANS data analysis, they explored that the swelling of the molecule occurs upon the accretion of the acid, notwithstanding the dependence of the conformation on the $\mathrm{pD}$ is about $4 \%$. An internal structural change of the dendrimer was found during protonation process. In 2008, Chen et al. studied using SANS the intramolecular cavity of G4-6 PAMAM dendrimer [21]. They found a significant amount of water inside the dendrimers. By changing the hydrogen-deuterium ratio of the solvent, obtaining the scattering contribution from the water and dendrimer components and combining it with an analytical model, the intramolecular space of PAMAM dendrimer was quantified. The internal cavity increases with the increase in the generation of dendrimers, while the water volume of a dendrimer remained invariant. In addition, they found the water density in dendrimers is higher than bulk water. Still in 2008, Chen et al. investigated the structure changes of the PAMAMs of different generations [23]. They found that with an obvious change of the intramolecular density profile, from neutral $\mathrm{pD}$ to acidic environment, the dendrimers' structure changes from a diffuse structure to a hard sphere, which means the molecular size remains invariant with a pD change. This finding suggests the PAMAM's molecular sizes have a weak dependence on the protonation effect. They also discovered a close relationship between generation and the degree-of-counterion-condensation. The chloride condensation is closely related to the higher generation of PAMAMs. In 2009, another paper by Chen et al. about the effect of counterion on the intermolecular structure and the properties of the conformation reported a surprising transformation of intra-molecular configuration with the use of $\mathrm{DBr}$ [27]. From fitting their data to the SANS model, they found out that the charged G5 PAMAM, which is protonated by $\mathrm{DBr}$, carried a less effective charge than that of $\mathrm{DCl}$ when $\alpha>1$. This work reveals that the specific counterion association is greater for the DBr G5 PAMAM than DCl G5 PAMAM. In other words, under the same level of molecular protonation, bromides have a much greater tendency to affiliate with the charged G5 PAMAM than chlorides. A similar conclusion was reached in another work from 2008, in which it was determined that the molecular size remains invariant with a $\mathrm{pD}$ change protonated by either $\mathrm{DBr}$ or DCl. Based on previous work, Chen et al. [23] continued studying the structural characteristics of PAMAMs by SANS. One year later, they illustrated the counterion valence effect on the $\mathrm{pH}$ responsiveness and its impact on the structure within a G5 PAMAM [28]. They discovered that the effective charge of PAMAMs is greatly reduced when they are associated with a large amount of divalent counterions; however, no obvious change of the intramolecular density was observed when the dendrimers were charged by $\mathrm{D}_{2} \mathrm{SO}_{4}$, conflicting with Chen's previous results for $\mathrm{DCl}$ charged 
dendrimers. The size of dendrimer molecules was validated, once again, as invariant with an increase in protonation. For G4 PAMAM, Chen et al. carried out a SANS experiment in 2010 [22]. They first provided the experimental results to verify an intramolecular structure change in the dendrimers core in order to show that the radial density profile decreases upon an increase in protonation, while the overall size remain the same. Using SANS in combination with SAXS, Chen et al. also investigated the structure characteristics of high-generation (G7 and G8) PAMAMs [29]. They reported an obvious molecular size increase along a continuous variation of the density profile of intramolecular structures with an increase in protonation. This work proposed a new picture of the structural characteristics of high generation PAMAMs and strongly supported the segmental back folding mechanism. This work opposed the current beliefs, which suggested the high generation PAMAMs are supposed to be crowded to hinder to local movement and electrostatic swelling. Most recently in 2011, Liu et al. investigated the G5 PAMAM in $\mathrm{D}_{2} \mathrm{O}$ with SANS, NMR, and PFG (proton pulsed filed gradient diffusion) [26]. In this work, NMR suggests a significant change of the hydrodynamic radius with increasing of protonation; however, the SANS results indicated little dependence between them, while displaying the transition from a dense core to a uniform hard sphere when the dendrimer is protonated by $\mathrm{DCl}$. The inconsistency in these results repudiates the validity of using the Stokes-Einstein relation to obtain the size of a polyelectrolyte dendrimer from dynamical measurements, although it is fairly accurate in determining the size of hard colloids.

Additionally, with the use of SAXS and SANS, Gröhn et al. studied the formation of gold nanoclusters inside dendrimer templates [30]. Maiti et al. studied the atomistic structure of ethylenediamine cored PAMAM family up through the 11th generation by SAXS and SANS [31]. By studying the G8 PAMAM by SANS, Nisato et al. found the dendrimer of a uniform sphere with a radius of gyration $R_{G}=4.0 \pm 0.15 \mathrm{~nm}$. They discovered the size is not dependent on the charge density or ionic strength of the solvent, which is contrary to the predictions of current models and simulations [32]. Liu et al. investigated the structural characteristics of G7 and G8 PAMAMs as a function of molecular protonation by SANS and SAXS. Their work revealed an increase of the molecular size and a continuous change in the intramolecular density profile [29]. Liu et al. also executed a study of the structural characterization for G5 PAMAMs by analyzing the SANS data [28].

\subsection{Characterization of Dendrimers by Quasi-Elastic Neutron Scattering (QENS)}

QENS is ideally suited to investigate both the segmental relaxation and local relaxations in polymer blends [15]. In a QENS experiment, the energies of the neutrons before and after the scattering event are differentiated, and the difference between them provides information on the time scale of motion within the sample. By combining the dominant cross-section of the macromolecules with the use of spectrometers having different energy resolutions, the local motion of macromolecules over a wide range of time-scales, from picoseconds to tens of nanoseconds, can be studied thoroughly.

QENS experiments measure the Incoherent Scattering Function (ISF $\omega)$. For dendrimers in solution, the ISF $\omega$ can be considered as the convolution of the dendrimer global, center-of-mass translational Brownian motion with its internal local motion [33]. In order to investigate the internal dynamics of a dendrimer, it is necessary to separate the local motion from the global translational diffusion using the following operation: 


$$
S_{\text {inc }}(Q, \omega)=N \times S_{\text {int }}(Q, \omega) \otimes S_{\text {trans }}(Q, \omega)+B(Q, \omega)
$$

where $S_{\text {inc }}(Q, \omega)$ is the total incoherent scattering function. $S_{\text {int }}(Q, \omega)$ is the contribution from internal motion, which will be convoluted with $S_{\text {trans }}(Q, \omega)$, the global translational diffusion. The Debye-Waller factor, sample scattering strength, and all the other factors corresponding to the normalization of the scattering intensity are collected into the parameter $N . B(Q, \omega)$ is the energy-dependent background that has been fitted with a linear function. $S_{\text {trans }}(Q, \omega)$ can be described by a Lorentzian $L_{\text {trans }}(Q, \omega)$ function with half-width at half-maximum (HWHM) of $\Gamma_{\text {trans }}(Q, \omega)=D_{s} Q^{2}$, where the self-diffusion coefficient $D_{s}$ can be obtained from NMR experiments. $S_{\text {int }}(Q, \omega)$ is the sum of an elastic contribution and a quasi-elastic Lorentzian contribution with an HWHM of $\Gamma_{\text {internal }}$, and $A_{0}(Q)$ here is the fraction of the elastic component that is $Q$-dependent.

$$
S_{\text {inc }}(Q, \omega)=A_{0}(Q) \times \delta(\omega)+\left(1-A_{0}(Q)\right) \times L_{\text {int }}(Q, \omega)
$$

Finally, the measured intensity $S_{\text {inc }}^{\exp }(Q, \omega)$, which is a convolution of $S_{\text {int }}(Q, \omega)$ with the instrument resolution function $R(Q, \omega)$, obtained from pure vanadium measurement, is represented as:

$$
\begin{aligned}
S_{\text {inc }}^{\text {exp }}(Q, \omega)=\{ & N \times\left[A_{0}(Q) \times \delta(\omega)+\left(1-A_{0}(Q)\right) \times L_{\text {int }}(Q, \omega)\right] \otimes L_{\text {trans }}(Q, \omega) \\
& +B(Q, \omega)\} \otimes R(Q, \omega)
\end{aligned}
$$

where $R(Q, \omega)$ is the resolution function of the instrument.

This data analysis methodology has been developed by Chen et al. and tested for PAMAMs in solutions of $\mathrm{DCl}$ and $\mathrm{D}_{2} \mathrm{O}$ at three different levels of $\mathrm{pH}$ values (molecular protonation levels $\alpha=0,1$, and 2) [34]. Figure 3 presents an example of the good agreement between QENS intensities and model fitting results [34].

Figure 3. (a) Quasi-elastic neutron scattering (QENS) intensity (blue symbols) recorded at $\mathrm{Q}=0.9 \AA^{-1}$, the total fitted curve (solid line), the corresponding components of translational diffusion (shaded area), the Lorentzian (dashed line) and background (dash-dotted line); (b) The dependence of the QENS intensity on $\alpha$ obtained at $Q=0.5 \AA^{-1}$ and (c) at $\mathrm{Q}=1.3 \AA^{-1}$ (Reproduced by permission of The Royal Society of Chemistry. http://pubs.rsc.org.libproxy.rpi.edu/en/Content/ArticleLanding/2011/SM/c0sm00671h).
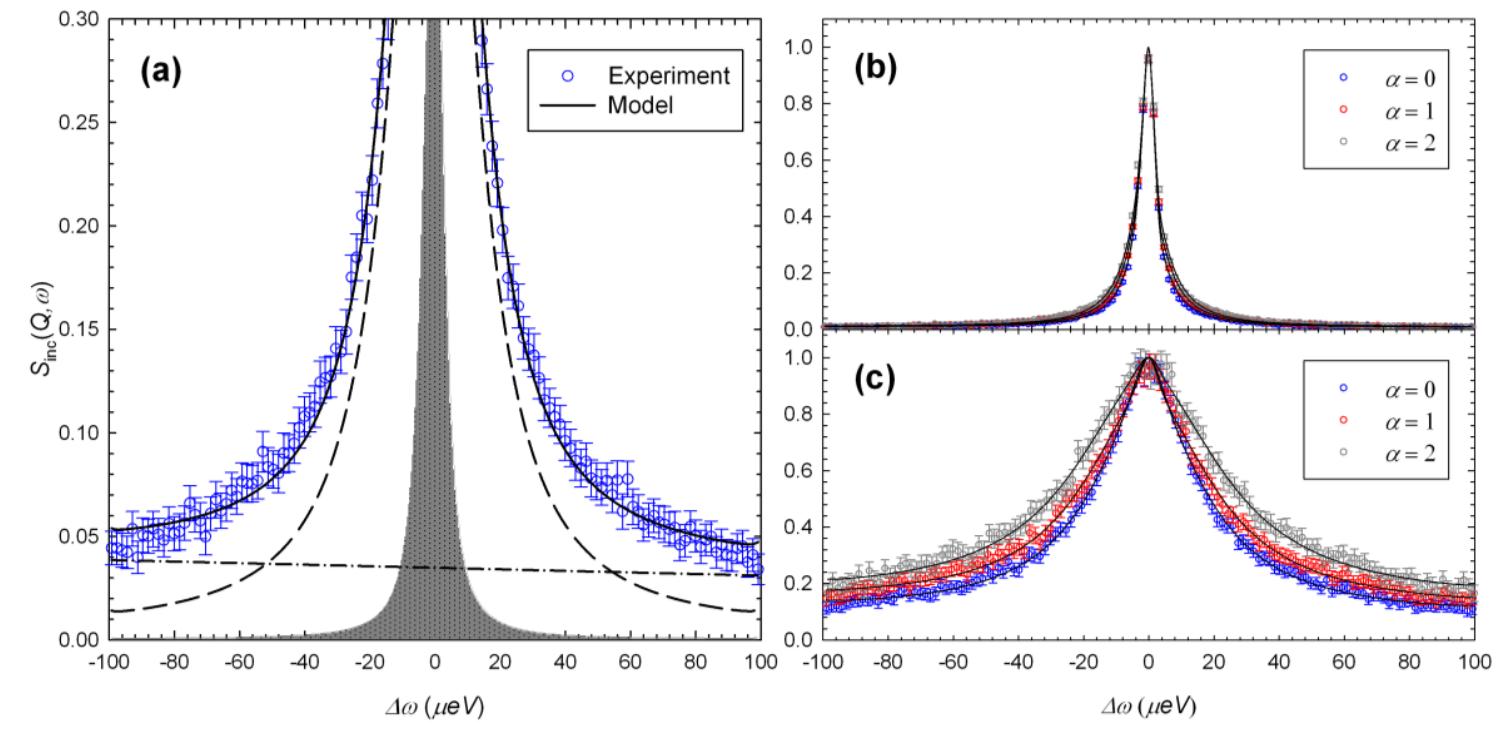
By combining QENS and high-resolution solution NMR spectroscopy, Chen et al. [34] first investigated the G5 PAMAMs as a function of protonation from a dynamical perspective and provided the first conclusive evidence from experimentation with $\mathrm{D}_{2} \mathrm{O}$. QENS clearly exhibits the local motion of dendrimer segments, illustrating an increased local motion with an increase in the molecular charge, which is contrary to the hypothesis that increased protonation will stiffen the dendrimer and hinder the local motion.

They used the diffusion coefficient $D$ and the sphere radius $R$ to describe the confined diffusive motion of dendrimers. QENS measurements show invariant $R$ at different protonation levels specified by $\alpha$ or pD values (Figure 4(b)) [34]. However, as seen from Figure 4(a) (the diffusion coefficient at different protonation levels: blue dots as the protonation level of $\alpha=0$, red dots as $\alpha=1$, and grey dots as $\alpha=2$ ), the diffusion coefficients of $\alpha=2$ are higher than that of $\alpha=1$, and the diffusion coefficients of $\alpha=1$ are higher than that of $\alpha=0$. This tells us despite the fluctuations at different $Q$ values, the internal diffusion coefficient $D$ is seen to increase steadily with the increase in dendrimer protonation [34].

Figure 4. The result of the QENS data analysis at different protonation levels: (a) the diffusion coefficient; (b) the radius of the sphere (Reproduced by permission of The Royal Society of Chemistry. http://pubs.rsc.org.libproxy.rpi.edu/en/Content/ArticleLanding/ 2011/SM/c0sm00671h).
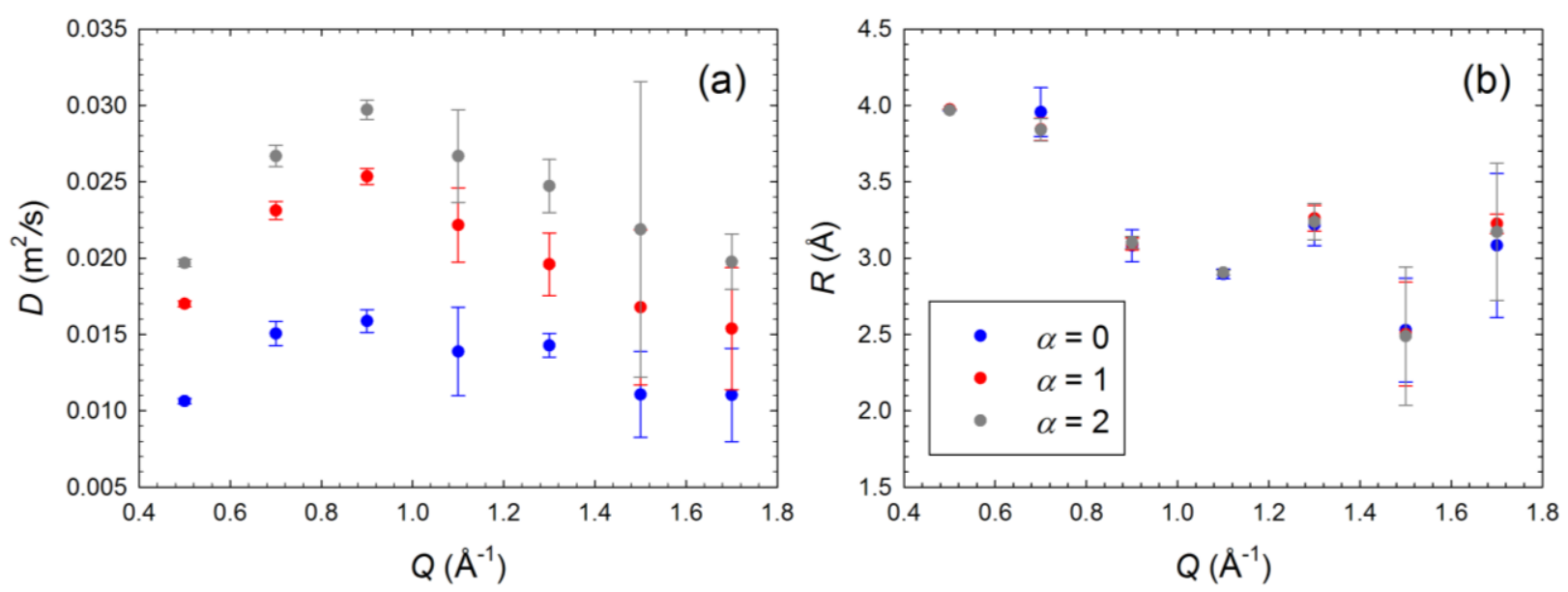

This tendency of $D$ demonstrates that the local diffusive motion is faster when the dendrimer is charged. It is believed that the induced protonation will result in the interaction with the counterions and water molecules. Additionally, intra-dendrimer space will be available for electrostatic swelling and mass redistribution inside the dendrimer.

\subsection{Characterization of Dendrimers by Small-Angle X-Ray Scattering (SAXS) and Light Scattering}

With the exception of SANS, dendrimers may be characterized by various techniques; the most wildly used scattering techniques include but are not limited to small angle X-ray scattering, small angle neutron scattering and light scattering.

Small-angle X-ray scattering (SAXS) and SANS data analysis are similar in many ways even though SANS uses neutrons and SAXS uses X-rays. For SAXS, the scattered X-ray signals by a given sample are recorded at very small angles. The signals within this angular range take the structural information of dendrimers to be explored. Prosa et al. used SAXS to characterize the single-particle 
scattering factors produced by different kinds of dendrimers [35]. They also examined the progression of intramolecular organizations of PAMAMs by SAXS and found an internal structure evolution from "star" to "sphere" organizations [36]. By using SAXS and neutron spin-echo spectroscopy Rathgeber et al. studied a single dendrimer for the dynamic and static structure factors and proposed a compact globular shape of dendrimers at least down to the G3 [37]. Mallamace et al. studied the internal density profile in a dendrimer system of various generations by SAXS, and proposed an internal self-similar structure for low generation dendrimers along with a spherical homogenous dense structure of higher generation [38].

Dynamic light scattering (DLS) is also widely used for studying the structure of macromolecules [36,38-42]. The measured data in DLS experiment are the correlation curve. Embodied within the correlation curve is all of the information regarding the diffusion of particles within the sample being measured. The diffusion coefficient (D) is calculated by fitting the correlation curve to an exponential function, with D being proportional to the lifetime of the exponential decay. Different mathematical approaches can be employed to determine D, such as: the cumulant method [43] and CONTIN algorithm [44]. D will then be used to calculate the hydrodynamic radius $\left(R_{H}\right)$ of dendrimers through the Stokes-Einstein equation.

$$
D=\frac{k T}{6 \pi \eta R_{H}}
$$

where $k$ is the Boltzmann constant, $T$ is the temperature, and $\eta$ is the medium viscosity. With small differences, the $R_{H}$ derived by DLS is comparable with $R_{G}$ in most cases. By using DLS Choi et al. obtained the mean diameter of PAMAM dendrimers to be around $200 \mathrm{~nm}$ [45]. Mourey et al. obtained the polyether dendrimers' molecular weight by Laser light scattering (LALLS), which agreed with the anticipated values determined from the synthetic method [46]. The following equation is used to obtain the average molecular weight from LALLS:

$$
\overline{M_{w}}=\frac{1}{m K} \int_{0}^{\infty} R_{\theta}(v) d v
$$

Here $R_{\theta}(v)$ is excess Rayleigh scattering at retention volume $v, K$ is an optical constant, and $\mathrm{m}$ is the total mass of sample [46].

\section{Application of Dendrimers as Anticancer Polymer Drug Carriers}

As one of the key candidates for a potential anticancer polymer drug carrier, dendrimers are nano-sized, radially symmetric molecules with well-defined, homogeneous and monodispersed structures consisting of tree-like arms or branches [6]. The flexible branches of a dendrimer contain voids that can physically trap and provide refuge for drug molecules from the outside environment [2]. Inclusion of hydrophobic drug molecules into dendrimers is typically accomplished by simple mixing of the polymer and drug solutions where the hydrophobic drug associates with the nonpolar core through hydrophobic interactions [47-50]. (Figure 5(a) [10]).

However, a sustained and controlled release of the encapsulated molecules in physiological solutions remains hard to achieve. For example, methotrexate (MTX) loaded into PEGylated dendrimers are released 10 times faster in an isotonic Tris buffer containing $150 \mathrm{mM} \mathrm{NaCl}$ solution than in a nonisotonic Tris buffer [49]. Similarly, there was a reported 70\% release of the MTX loaded 
into G5-MTX inclusion complexes during an incubation period of 2.5 hours in phosphate buffered saline (PBS). In comparison, there was an insignificant MTX release in water under the same experimental conditions [48].

Figure 5. (a) Schematic drawing of a dendrimer carrier encapsulating hydrophobic drug molecules in the dendrimer's voids to increase their aqueous solubility and control their release rate; (b) Schematic drawing showing a dendrimer-drug conjugate where the drug molecules (red ovals) are either directly coupled (solid lines) to dendrimer's surface groups or are coupled via a $\mathrm{pH}$ sensitive linkage (blue rectangle) (Reprinted (adapted) with permission from Medina, S.H. and El-Sayed, M.E., Dendrimers as carriers for delivery of chemotherapeutic agents. Chem Rev., 2009. 109 (7): p. 3141-57. Copyright 2012 American Chemical Society).

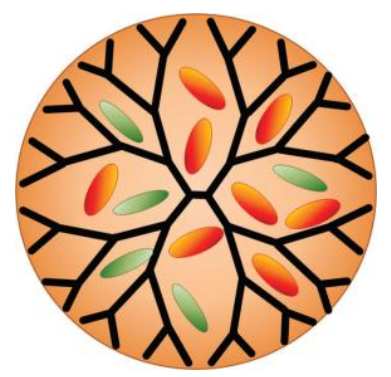

(a)

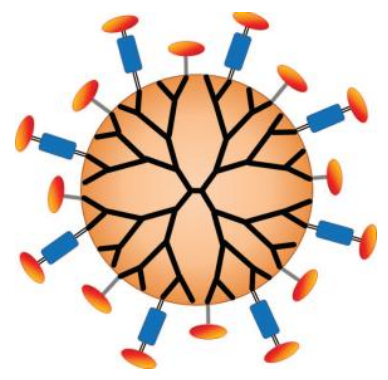

(b)

Covalent conjugation of anticancer drugs to the dendrimer's surface groups has been used to achieve a controlled spatial and temporal release of the attached drugs [3]. The large number of the dendrimer's surface groups and the versatility in their chemical structures allow the conjugation of different anticancer drugs, imaging agents, and/or targeting ligands, while maintaining the dendrimer's compact spherical geometry in solution (Figure 5(b) [10]). PAMAM-doxorubicin (PAMAM-DOX) conjugates exhibited 5-fold lower toxicity toward HeLa cells upon incubation with $0.001-1,000 \mu \mathrm{M}$ DOX equivalent for $24 \mathrm{~h}$. In comparison, free DOX resulted in an insignificant drug release $(<5 \%$ of DOX is released upon incubation in PBS solution for $24 \mathrm{~h})$ [51].

Among the family of dendrimers, PAMAMs were the first synthesized and commercially available group. They have been considered for development as delivery agents for a wide spectrum of guest materials in aqueous environments [52-54]. For example, anticancer drugs, such as Cisplatin, Dimethoxycurcumin, Doxorubicin, Etoposide, 5-Fluorouracil, Methotrexate, are soluble in dendrimers, and therefore can be encapsulated in dendrimers forming Dendrimer-Drug Complexes [55]. Additionally, anticancer drugs such as paclitaxel (Taxol) can be conjugated to PAMAM dendrimers via a succinic acid linker, also forming Dendrimer-Drug Conjugates [55,56].

Despite the promise of dendrimer-based drug delivery systems, their translation into actual cancer therapies with defined dosing regimen is lagging. This can be partially attributed to the regulatory hurdles that demand detailed characterization of these polymeric carriers and the incorporated drugs [10]. Moreover, controlling the release kinetics of the encapsulated or conjugated drug remains a challenging task that depends on a number of factors, such as: the type (hydrophobicity, size, etc.) of the drug, the generation number of the dendritic carrier, and the type and extent of the modification of 
the dendrimer's interface with drug and water due to external environments. For example, the impact of either encapsulation (Figure 5(a)) or conjugation (Figure 5(b)) of mostly hydrophobic anticancer drugs to the hydrophilic dendrimer surfaces has not been fully investigated at the microscopic level. Indeed, a concern is that the active drug would "leak" out prematurely, thereby reducing the amount available for the intended therapeutic intervention, or more ominously, resulting in systemic toxicity [6]. This is significant since many anticancer drugs are hydrophobic, and as such, their presence is likely to change the surface and interfacial characteristics, and as a result, the in vivo behavior of the dendrimer carriers. Even without drugs, the solution's $\mathrm{pH}$ has a significant impact on the structure of dendrimers [57].

\section{Conclusions}

Dendrimer research and analysis have progressed tremendously during the past few decades. Due to their highly controllable structures, dendrimers are a promising candidate for medical uses, such as cancer diagnosis and treatment, such as drug delivery methods, and as therapeutic agents. Scattering techniques, such as SANS, QENS, SAXS, and DLS are of essential means to study the interfacial behaviors of dendrimer systems at the nano-scale level. These techniques can give important information on the shape, structure, conformation, and dynamics of dendrimers. They are of great importance to improve our understanding of dendrimers, enhance new therapeutic design, clinical diagnostics and assays. In this paper, we present the scattering studies associated with PAMAMs. Similar studies may be extended to other kind of dendrimers. Although there is a long way before the medical uses of dendrimers come into fruition, extensive research is underway in an effort to realize Dendrimer-Drug Complex and Dendrimer-Drug Conjugate solutions. The biomedical application of dendrimers is a clear indication about the optimistic future of this class of polymers.

\section{Acknowledgments}

XW, BW, and LL were supported in part by U.S. Department of Energy, under NERI-C Award No. DE-FG07-07ID14889, and US Nuclear Regulatory Commission, under Award No. NRC-38-08-950. WRC acknowledges the support of The Scientific User Facilities Division, Office of Basic Energy Sciences, US Department of Energy for Oak Ridge National Laboratory's Spallation Neutron Source and High Flux Isotope Reactor.

\section{References}

1. Garg, T.; Singh, O.; Arora, S.; Murphy, R. Dendrimer-A novel scaffold for drug delivery. Int. J. Pharm. Sci. Rev. Res. 2011, 7, 211-220.

2. Dykes, G.M. Dendrimers: A review of their appeal and applications. J. Chem. Technol. Biotechnol. 2001, 76, 903-918.

3. Gohel, M.C.; Parikh, R.K.; Bariya, S.H.; Nagori, S.A.; Gandhi, A.; Patel, V.; Patel, T.; Pandya, R.; Kharadi, S.; Patel, P.; et al. Dendrimer: An overview. Pharm. Rev. Target. Drug Deliv. Syst. 2009, 7, Available online: http://www.pharmainfo.net/reviews/dendrimer-overview (accessed on 21 February 2012). 
4. Ballauff, M.; Likos, C.N. Dendrimers in solution: Insight from theory and simulation. Angew. Chem. Int. Ed. 2004, 43, 2998-3020.

5. Fréchet, J.M.J.; Tomalia, D.A. Dendrimers and other Dendritic Polymers; Wiley: New York, NY, USA, 2001.

6. Sampathkumar, S.-G.; Yarema, K.J. Dendrimers for cancer treatment and diagnosis. In Nanomaterials for Cancer Diagnosis, Kumar, C.S.S.R., Ed.; Nanotechnologies for the Life Sciences; Wiley-VCH Verlag GmbH \& Co. KGaA: Weinheim, Germany, 2007; Volume 7.

7. Dykes, G.M. Dendrimers: A review of their appeal and applications. J. Chem. Technol. Biotechnol. 2001, 76, 903-918.

8. Tomalia, D.A.; Baker, H.; Dewald, J.; Hall, M.; Kallos, G.; Martin, S.; Roeck, J.; Ryder, J.; Smith, P. A new class of polymers-Starburst-dendritic macromolecules. Polym. J. 1985, 17, $117-132$.

9. Tomalia, D.A.; Naylor, A.M.; Goddard, W.A. Starburst dendrimers: molecular-level control of size, shape, surface chemistry, topology, and flexibility from atoms to macroscopic matter. Angew. Chem. Int. Ed. Eng. 1990, 29, 138-175.

10. Medina, S.H.; El-Sayed, M.E. Dendrimers as carriers for delivery of chemotherapeutic agents. Chem. Rev. 2009, 109, 3141-3157.

11. Liu, Y.; Chen, C.-Y.; Chen, H.-L.; Hong, K.; Shew, C.-Y.; Li, X.; Liu, L.; Melnichenko, Y.B.; Smith, G.S.; Herwig, K.W.; et al. Electrostatic swelling and conformational variation observed in high-generation polyelectrolyte dendrimers. J. Phys. Chem. Lett. 2010, 1, 2020-2024.

12. Pitzer, K.S. Activity Coefficients in Electrolyte Solutions; CRC Press: Boca Raton, FL, USA, 1991.

13. Chen, W.R.; Porcar, L.; Liu, Y.; Butler, P.D.; Magid, L.J. Small angle neutron scattering studies of the counterion effects on the molecular conformation and structure of charged g4 PAMAM dendrimers in aqueous solutions. Macromolecules 2007, 40, 5887-5898.

14. Liu, Y.; Porcar, L.; Hong, K.L.; Shew, C.Y.; Li, X.; Liu, E.; Butler, P.D.; Herwig, K.W.; Smith, G.S.; Chen, W.R. Effect of counterion valence on the $\mathrm{pH}$ responsiveness of polyamidoamine dendrimer structure. J. Chem. Phys. 2010, 132, 124901.

15. Maranas, J.K. The effect of environment on local dynamics of macromolecules. Curr. Opin. Colloid Interface Sci. 2007, 12, 29-42.

16. Lindner, P.; Zemb, T. Neutron, X-Ray and Light Scattering: Introduction to an Investigate Tool for Colloidal and Polymeric Systems; Elsevier: Amsterdam, The Netherlands, 1991.

17. Li, X.; Hong, K.; Liu, Y.; Shew, C.-Y.; Liu, L.; Herwig, K.W.; Smith, G.S.; Zhao, J.; Zhang, G.; Pispas, S.; Chen, W.-R. Water distributions in PS-b-P(S-g-PEO) block grafted copolymers aggregates in aqueous solutions revealed by contrast variation SANS study. J. Chem. Phys. 2010, $133,144912$.

18. Kline, S.R. Reduction and analysis of SANS and USANS data using IGOR Pro. J. Appl. Cryst. 2006, 39, 895-900.

19. Laurati, M.; Stellbrink, J.; Lund, R.; Willner, L.; Zaccarelli, E.; Richter, D. Asymmetric poly(ethylene-alt-propylene)-poly(ethylene oxide) micelles: A system with starlike morphology and interactions. Phys. Rev. E 2007, 76, 041503.

20. Smit, B.; Frenkel, D. Understanding Molecular Simulation; Academic Press: Amsterdam, The Netherlands, 2001. 
21. Li, T.F.; Hong, K.; Porcar, L.; Verduzco, R.; Butler, P.D.; Smith, G.S.; Liu, Y.; Chen, W.R. Assess the intramolecular cavity of a PAMAM dendrimer in aqueous solution by small-angle neutron scattering. Macromolecules 2008, 41, 8916-8920.

22. Porcar, L.; Hong, K.L.; Butler, P.D.; Herwig, K.W.; Smith, G.S.; Liu, Y.; Chen, W.R. Intramolecular structural change of PAMAM dendrimers in aqueous solutions revealed by small-angle neutron scattering. J. Phys. Chem. B 2010, 114, 1751-1756.

23. Porcar, L.; Liu, Y.; Verduzco, R.; Hong, K.L.; Butler, P.D.; Magid, L.J.; Smith, G.S.; Chen, W.R. Structural INVESTIGATION OF PAMAM dendrimers in aqueous solutions using small-angle neutron scattering: Effect of generation. J. Phys. Chem. B 2008, 112, 14772-14778.

24. Hansen, J.-P.; McDonald, I.R. Theory of Simple Liquids, 3rd ed.; Academic Press: Amsterdam, The Netherlands, 2006.

25. Chen, S.-H.; Sheu, E.Y. Micellar Solutions and Microemulsions: Structure, Dynamics, and Statistical Thermodynamics; Springer: New York, NY, 1990.

26. Hong, K.; Liu, Y.; Porcar, L.; Liu, D.; Gao, C.Y.; Smith, G.S.; Herwig, K.W.; Cai, S.; Li, X.; $\mathrm{Wu}, \mathrm{B}$; et al. Structural response of polyelectrolyte dendrimer towards molecular protonation: The inconsistency revealed by SANS and NMR. J. Phys.: Condens. Matter 2011, 24, 064116.

27. Porcar, L.; Liu, Y.; Hong, K.L.; Butler, P.D.; Huang, E.W.; Chen, W.R. Counterion association and structural conformation change of charged PAMAM dendrimer in aqueous solutions revealed by small angle neutron scattering. Macromol. Symp. 2009, 279, 65-71.

28. Liu, Y.; Porcar, L.; Hong, K.L.; Shew, C.Y.; Li, X.; Liu, E.; Butler, P.D.; Herwig, K.W.; Smith, G.S.; Chen, W.R. Effect of counterion valence on the $\mathrm{pH}$ responsiveness of polyamidoamine dendrimer structure. J. Chem. Phys. 2010, 132, 124901.

29. Liu, Y.; Chen, C.Y.; Chen, H.L.; Hong, K.L.; Shew, C.Y.; Li, X.; Liu, L.; Melnichenko, Y.B.; Smith, G.S.; Herwig, K.W.; et al. Electrostatic swelling and conformational variation observed in high-generation polyelectrolyte dendrimers. J. Phys. Chem. Lett. 2010, 1, 2020-2024.

30. Grohn, F.; Bauer, B.J.; Akpalu, Y.A.; Jackson, C.L.; Amis, E.J. Dendrimer templates for the formation of gold nanoclusters. Macromolecules 2000, 33, 6042-6050.

31. Maiti, P.K.; Cagin, T.; Wang, G.F.; Goddard, W.A. Structure of PAMAM dendrimers: Generations 1 through 11. Macromolecules 2004, 37, 6236-6254.

32. Nisato, G.; Ivkov, R.; Amis, E.J. Size invariance of polyelectrolyte dendrimers. Macromolecules 2000, 33, 4172-4176.

33. Zanotti, J.M.; Bellissent-Funel, M.C.; Parello, J. Hydration-coupled dynamics in proteins studied by neutron scattering and NMR: The case of the typical EF-hand calcium-binding parvalbumin. Biophys. J. 1999, 76, 2390-2411.

34. Li, X.; Zamponi, M.; Hong, K.; Porcar, L.; Shew, C.-Y.; Jenkins, T.; Liu, E.; Smith, G.S.; Herwig, K.W.; Liu, Y.; Chen, W.-R. pH Responsiveness of polyelectrolyte dendrimers: a dynamical perspective. Soft Matter 2011, 7, 618-622.

35. Prosa, T.J.; Bauer, B.J.; Amis, E.J.; Tomalia, D.A.; Scherrenberg, R. A SAXS study of the internal structure of dendritic polymer systems. J. Polym. Sci. Part B Polym. Phys. 1997, 35, 2913-2924.

36. Prosa, T.J.; Bauer, B.J.; Amis, E.J. From stars to spheres: A SAXS analysis of dilute dendrimer solutions. Macromolecules 2001, 34, 4897-4906. 
37. Rathgeber, S.; Monkenbusch, M.; Kreitschmann, M.; Urban, V.; Brulet, A. Dynamics of star-burst dendrimers in solution in relation to their structural properties. J. Chem. Phys. 2002, 117, 4047-4062.

38. Mallamace, F.; Canetta, E.; Lombardo, D.; Mazzaglia, A.; Romeo, A.; Scolaro, L.M.; Maino, G. Scaling properties in the internal structure of dendrimer systems. Physica A 2002, 304, 235-243.

39. Kucerka, N.; Nagle, J.F.; Sachs, J.N.; Feller, S.E.; Pencer, J.; Jackson, A.; Katsaras, A.J. Lipid bilayer structure determined by the simultaneous analysis of neutron and X-ray scattering data. Biophys. J. 2008, 95, 2356-2367.

40. Jeng, U.; Lin, T.L.; Hu, Y.; Lin, J.M.; Huang, Y.S.; Liang, K.S.; Fan, L.; Thiyagarajan, P. Complex structure of fullerene star ionomers and sodium dodecyl sulfate resolved by contrast variation with SANS and SAXS. Nucl. Instrum. Methods Phys. Res. Sect. A 2009, 600, 294-296.

41. He, J.B.; Niu, Z.W.; Tangirala, R.; Wan, J.Y.; Wei, X.Y.; Kaur, G.; Wang, Q.; Jutz, G.; Boker, A.; Lee, B.; et al. Self-assembly of tobacco mosaic virus at oil/water interfaces. Langmuir 2009, 25, 4979-4987.

42. Jana, C.; Jayamurugan, G.; Ganapathy, R.; Maiti, P.K.; Jayaraman, N.; Sood, A.K. Structure of poly(propyl ether imine) dendrimer from fully atomistic molecular dynamics simulation and by small angle x-ray scattering. J. Chem. Phys. 2006, 124, 204719.

43. Frisken, B.J. Revisiting the method of cumulants for the analysis of dynamic light-scattering data. Appl. Opt. 2001, 40, 4087-4091.

44. Provencher, S.W. CONTIN: A general purpose constrained regularization program for inverting noisy linear algebraic and integral equations. Comp. Phys. Commun. 1982, 27, 229-242.

45. Choi, J.S.; Nam, K.; Park, J.; Kim, J.B.; Lee, J.K.; Park, J. Enhanced transfection efficiency of PAMAM dendrimer by surface modification with L-arginine. J. Control. Release 2004, 99, 445-456.

46. Mourey, T.H.; Turner, S.R.; Rubinstein, M.; Frechet, J.M.J.; Hawker, C.J.; Wooley, K.L. Unique behavior of dendritic macromolecules-Intrinsic-viscosity of polyether dendrimers. Macromolecules 1992, 25, 2401-2406.

47. Goodwin, A.P.; Lam, S.S.; Frechet, J.M. Rapid, efficient synthesis of heterobifunctional biodegradable dendrimers. J. Am. Chem. Soc. 2007, 129, 6994-6995.

48. Patri, A.K.; Kukowska-Latallo, J.F.; Baker, J.R., Jr. Targeted drug delivery with dendrimers: comparison of the release kinetics of covalently conjugated drug and non-covalent drug inclusion complex. Adv. Drug Del. Rev. 2005, 57, 2203-2214.

49. Kojima, C.; Kono, K.; Maruyama, K.; Takagishi, T. Synthesis of polyamidoamine dendrimers having poly(ethylene glycol) grafts and their ability to encapsulate anticancer drugs. Bioconjug. Chem. 2000, 11, 910-917.

50. Morgan, M.T.; Carnahan, M.A.; Immoos, C.E.; Ribeiro, A.A.; Finkelstein, S.; Lee, S.J.; Grinstaff, M.W. Dendritic molecular capsules for hydrophobic compounds. J. Am. Chem. Soc. 2003, 125, 15485-15489.

51. Kono, K.; Kojima, C.; Hayashi, N.; Nishisaka, E.; Kiura, K.; Watarai, S.; Harada, A. Preparation and cytotoxic activity of poly(ethylene glycol)-modified poly(amidoamine) dendrimers bearing adriamycin. Biomaterials 2008, 29, 1664-1675.

52. Fischer, M.; Vögtle, F. Dendrimers: From design to application-A progress report. Angew. Chem. Int. Ed. 1999, 38, 884-905. 
53. Esfand, R.; Tomalia, D.A. Poly(amidoamine) (PAMAM) dendrimers: From biomimicry to drug delivery and biomedical applications. Drug Discovery Today 2001, 6, 427-436.

54. Boas, U.; Heegaard, P.M.H. Dendrimers in drug research. Chem. Soc. Rev. 2004, 33, 43-63.

55. Astruc, D.; Boisselier, E.; Ornelas, C. Dendrimers designed for functions: From physical, photophysical, and supramolecular properties to applications in sensing, catalysis, molecular electronics, photonics, and nanomedicine. Chem. Rev. 2010, 110, 1857-1959.

56. Majoros, I.J.; Myc, A.; Thomas, T.; Mehta, C.B.; Baker, J.R. PAMAM dendrimer-based multifunctional conjugate for cancer therapy: Synthesis, characterization, and functionality. Biomacromolecules 2006, 7, 572-579.

57. Maiti, P.K.; Cagin, T.; Lin, S.T.; Goddard, W.A. Effect of solvent and $\mathrm{pH}$ on the structure of PAMAM dendrimers. Macromolecules 2005, 38, 979-991.

(C) 2012 by the authors; licensee MDPI, Basel, Switzerland. This article is an open access article distributed under the terms and conditions of the Creative Commons Attribution license (http://creativecommons.org/licenses/by/3.0/). 